\title{
Size-dependent $\pi_{\mathrm{g}}+\pi_{\mathrm{u}}$ Combination Band Intensities of Polyynes $\mathrm{C}_{2 n} \mathrm{H}_{2}(n=1-9)$ Analyzed by the Local $\mathrm{CCH}$ Bending and the Linear Response Functions.
}

\author{
Masafumi Tsuyuki, ${ }^{1)}$ Yuto Kugaya, ${ }^{1)}$ Hideto Kanamori, ${ }^{2)}$ and Satoshi Yabushita ${ }^{1)}$ \\ ${ }^{1}$ Department of Chemistry, Faculty of Science and Technology, Keio University, 3-14-1 Hiyoshi, Kohoku- \\ ku, Yokohama, 223-8522 Japan \\ ${ }^{2}$ Department of Physics, Tokyo Institute of Technology, Ohokayama 2-12-1, Tokyo 152-8551, Japan
}

\section{Supporting Information}

(S1) Optimized Geometries

Table S1. Symmetry unique bond lengths $(\AA)$ of the liner $\mathrm{C}_{2 n} \mathrm{H}_{2}$ optimized by CAM-B3LYP/6$311++G(3 d, 3 p)$.

\begin{tabular}{|c|c|c|c|c|c|c|c|c|c|}
\hline & $n=1$ & $n=2$ & $n=3$ & $n=4$ & $n=5$ & $n=6$ & $n=7$ & $n=8$ & $n=9$ \\
\hline$R(1 \mathrm{H}-1 \mathrm{C})$ & 1.061737 & 1.061393 & 1.061523 & 1.061625 & 1.061691 & 1.061739 & 1.061775 & 1.061803 & 1.061784 \\
\hline$R(2 \mathrm{C} \equiv 3 \mathrm{C})$ & 1.196285 & 1.204498 & 1.207108 & 1.208066 & 1.208494 & 1.208699 & 1.208806 & 1.208860 & 1.208931 \\
\hline$R(3 \mathrm{C}-4 \mathrm{C})$ & & 1.364458 & 1.355139 & 1.352194 & 1.350938 & 1.350294 & 1.349957 & 1.349750 & 1.349606 \\
\hline$R(4 \mathrm{C} \equiv 5 \mathrm{C})$ & & & 1.214854 & 1.218560 & 1.220083 & 1.220819 & 1.221202 & 1.221411 & 1.221502 \\
\hline$R(5 \mathrm{C}-6 \mathrm{C})$ & & & & 1.343731 & 1.339836 & 1.338103 & 1.337197 & 1.336695 & 1.336427 \\
\hline$R(6 \mathrm{C} \equiv 7 \mathrm{C})$ & & & & & 1.222844 & 1.224698 & 1.225622 & 1.226111 & 1.226374 \\
\hline$R(7 \mathrm{C}-8 \mathrm{C})$ & & & & & & 1.335444 & 1.333441 & 1.332347 & 1.331794 \\
\hline$R(8 \mathrm{C} \equiv 9 \mathrm{C})$ & & & & & & & 1.226748 & 1.227778 & 1.228321 \\
\hline$R(9 \mathrm{C}-10 \mathrm{C})$ & & & & & & & & 1.331244 & 1.330106 \\
\hline$R(10 \mathrm{C} \equiv 11 \mathrm{C})$ & & & & & & & & & 1.228861 \\
\hline
\end{tabular}

\section{(S2) Numerical Consistency of the Calculated $M_{2}$ Parameters}

In the present work, the $M_{2}$ coefficients were first determined globally through the least-squares fit of sixthorder polynomial DMFs to the single-point calculated $\mu_{z}$ data at $\theta_{x}=0-90^{\circ}$ as shown in Figures 3 and 4 . These coefficients were analyzed using $\rho^{(2)}(\boldsymbol{r})$, which are the local differential quantities calculated by the linear response function. Thus we are actually comparing two rather different quantities. Here we show the numerical consistency of this computational strategy.

The $M_{2}$ values determined by the above least-squares fit in Figure 4 are also given in Table S2 below under the name of "Single-point calculations" and "Global fit". Those in "Local differentiation" were obtained by numerical differentiation using two $\mu_{z}$ data around the equilibrium geometry $\left(\theta_{x}= \pm 5^{\circ}\right)$. These two kinds of $M_{2}$ parameters in Table S2 are very close to each other. Therefore, in these polyynes, a meaningful discussion is possible by local differentiation approaches.

Since our main objective is to investigate the physical origin which determines the size dependence of the $M_{2}$ values, we first evaluated $\partial \rho(\boldsymbol{r}) / \partial \theta_{x}$ in Figure 5a using eq 25 and the external potential change $\delta v\left(\boldsymbol{r}^{\prime}\right)$ in eq. 27. These $M_{1}$ and $\partial \rho(\boldsymbol{r}) / \partial \theta_{x}$ values have been calculated analytically using the so-called response vectors at $\theta_{x}= \pm \Delta \theta_{x}= \pm 5^{\circ}= \pm 5 \times \frac{\pi}{180}$ radian 


$$
M_{1}=R_{\mathrm{CH}} \cos \theta_{x}+4 \sum_{a i}\left\langle\varphi_{a}|z| \varphi_{i}\right\rangle \sum_{b j}\left(\mathcal{A}^{-1}\right)_{a i, b j}\left\langle\varphi_{b}\left|\frac{\partial v}{\partial \theta_{x}}\right| \varphi_{j}\right\rangle
$$

Then, we have evaluated $M_{2}$ in Table 1 and $\partial^{2} \rho(\boldsymbol{r}) / \partial \theta_{x}{ }^{2}$ in Figure $5 \mathrm{~b}$ by the central difference using the analytically obtained first derivatives at $\left(\theta_{x}, \theta_{y}\right)=\left( \pm \Delta \theta_{x}, 0\right)$

$$
\begin{aligned}
& M_{2}=\frac{1}{2} \frac{M_{1}\left(\theta_{x}=\Delta \theta_{x}\right)-M_{1}\left(\theta_{x}=-\Delta \theta_{x}\right)}{2 \Delta \theta_{x}} \\
& \frac{\partial^{2} \rho(\boldsymbol{r})}{\partial \theta_{x}^{2}}=\frac{\left[\frac{\partial \rho(\boldsymbol{r})}{\partial \theta_{x}}\right]_{\theta_{x}=\Delta \theta_{x}}-\left[\frac{\partial \rho(\boldsymbol{r})}{\partial \theta_{x}}\right]_{\theta_{x}=-\Delta \theta_{x}}}{2 \Delta \theta_{x}}
\end{aligned}
$$

The first factor $1 / 2$ in eq S2 above comes from our definition of eq 18 in the main text. These $M_{2}$ values given in the row of "Linear response function" in Table S2 are in good agreement with the above two kinds of data by "Single-point calculations". Those obtained with eq 18 by numerical integrations with the $z \rho^{(2)}(\boldsymbol{r})$ were also given in the row of "Numerical integration" in Table S2. Again, we see good overall numerical accuracy. It is concluded that the numerical consistency of these $M_{2}$ values is good enough to justify the whole theoretical methods used in the present study.

The second derivatives of electron density can be calculated as the second-order numerical differentiations of the electron density $\rho(\boldsymbol{r})$, but the use of the linear response function to analytically compute $\partial \rho(\boldsymbol{r}) / \partial \theta_{x}$ is more advantageous from the viewpoint of numerical accuracy.

Table S2. The $M_{2}$ values (debye radian ${ }^{-2}$ ) of $\mathrm{C}_{2 n} \mathrm{H}_{2}$ by the curvilinear displacements.

\begin{tabular}{c|c|ccccccccc}
\hline \multicolumn{2}{c|}{ Method } & $n=1$ & $n=2$ & $n=3$ & $n=4$ & $n=5$ & $n=6$ & $n=7$ & $n=8$ & $n=9$ \\
\hline $\begin{array}{c}\text { Single-point } \\
\text { calculations }\end{array}$ & $\begin{array}{c}\text { Global fit } \\
\text { Local differentiation of } \\
\mu_{z}\end{array}$ & -1.104 & -1.507 & -1.785 & -2.010 & -2.200 & -2.363 & -2.502 & -2.622 & -2.726 \\
\hline $\begin{array}{c}\text { Linear } \\
\text { response } \\
\text { function }\end{array}$ & $\begin{array}{c}\text { Numerical derivative of } \\
\text { analytical } M_{1}\end{array}$ & -1.111 & -1.495 & -1.780 & -2.002 & -2.198 & -2.361 & -2.504 & -2.625 & -2.731 \\
\hline $\begin{array}{c}\text { Numerical integration } \\
\text { of } z \rho^{(2)}(\boldsymbol{r})\end{array}$ & -1.116 & -1.507 & -1.792 & -2.011 & -2.201 & -2.358 & -2.496 & -2.620 & -2.725 \\
\hline
\end{tabular}

${ }^{a}$ Numerical integration with a grid spacing of 0.1 bohr covering the molecular region with the additional range of \pm 6 bohr from the outer atoms. 
Table S3. Frequencies $\left(\mathrm{cm}^{-1}\right)$ and intensities $\left(\mathrm{km} \mathrm{mol}^{-1}\right)$ of the $\mathrm{C}_{2} \mathrm{H}_{2}$ fundamental and combination bands.

\begin{tabular}{c|cccccc}
\hline & \multicolumn{2}{|c}{ This work } & \multicolumn{2}{c}{ VPT2 $^{\mathrm{a}}$} & \multicolumn{2}{c}{ Experimental } \\
& (CAM-B3LYP/6-311++G(3d,3p) $)$ & \multicolumn{2}{c}{$(\mathrm{CCSD}(\mathrm{T}) / \mathrm{ANO} 1)$} & & \\
\hline & Frequency & Intensity & Frequency & Intensity & Frequency & Intensity \\
\hline$v_{3}\left(\sigma_{\mathrm{u}}{ }^{+}\right)$ & $3307.0^{\mathrm{b}}$ & $96.0^{\mathrm{b}}$ & 3285.9 & 74.8 & $3288.6^{\mathrm{c}}$ & $71.1 \pm 2.3^{\mathrm{d}}$ \\
$v_{4}\left(\pi_{\mathrm{g}}\right)$ & $687.2^{\mathrm{e}}$ & 0 & 600.6 & 0 & $612.9^{\mathrm{f}}$ & 0 \\
$v_{5}\left(\pi_{\mathrm{u}}\right)$ & $772.2^{\mathrm{e}}$ & $199.4^{\mathrm{e}}$ & 734.7 & $183.4^{\mathrm{h}}$ & $730.3^{\mathrm{c}}$ & $175 \pm 0.5^{\mathrm{d}}$ \\
$v_{4}+v_{5}\left(\Sigma_{\mathrm{u}}^{+}\right)$ & $1340.4^{\mathrm{g}}$ & $18.8^{\mathrm{g}}$ & 1329.2 & $21.6^{\mathrm{f}}$ & $1328.1^{\mathrm{c}}$ & $22.3 \pm 0.4^{\mathrm{d}}$ \\
$v_{1}+v_{3}\left(\Sigma_{\mathrm{u}}^{+}\right)$ & $6515.8^{\mathrm{b}}$ & $1.6^{\mathrm{b}}$ & 6928.7 & 2.0 & $6556.5^{\mathrm{i}}$ & $1.6^{\mathrm{i}}$ \\
\hline
\end{tabular}

${ }^{\text {a }}$ Calculated values from ref. 17. ${ }^{\mathrm{b}}$ Calculated values by the Local mode model with the single $\mathrm{CH}$ stretching

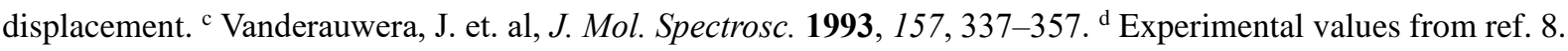
${ }^{\mathrm{e}}$ Calculated values by the normal mode model. ${ }^{\mathrm{f}}$ Experimental values from Plíva, J. Journal of Molecular Spectroscopy 1972, 44, 165-182. ${ }^{\mathrm{g}}$ Calculated values by the Local mode model with the single CCH angle. ${ }^{\mathrm{h}}$ The value from ref. 17 was multiplied by two for the degenerate $\pi_{\mathrm{u}}$ mode. ${ }^{\mathrm{i}}$ El Hachtouki, R.; Auwera, J. V. J. Mol. Spectrosc. 2002, 216, 355-362.

Table S4. Frequencies $\left(\mathrm{cm}^{-1}\right)$ and intensities $\left(\mathrm{km} \mathrm{mol}^{-1}\right)$ of the $\mathrm{C}_{8} \mathrm{H}_{2}$ fundamental and combination bands.

\begin{tabular}{|c|c|c|c|c|c|c|}
\hline & \multicolumn{2}{|c|}{$\begin{array}{c}\text { This work } \\
(\text { CAM-B3LYP/6-311++G(3d,3p)) }\end{array}$} & \multicolumn{2}{|c|}{$\begin{array}{c}\mathrm{VPT}^{\mathrm{a}} \\
(\mathrm{CCSD}(\mathrm{T}) / \mathrm{ANO} 1)\end{array}$} & \multicolumn{2}{|c|}{ Experimental $^{\mathrm{b}}$} \\
\hline & Frequency & Intensity & Frequency & Intensity & Frequency & Intensity \\
\hline$v_{6}\left(\sigma_{\mathrm{u}}^{+}\right)$ & $3311.2^{\mathrm{c}}$ & $265.9^{c}$ & 3328.8 & 214.2 & 3329.4 & $146 \pm 29$ \\
\hline$v_{10}\left(\pi_{\mathrm{g}}\right)$ & $709.9^{d}$ & $0^{\mathrm{d}}$ & 620.0 & 0 & - & - \\
\hline$v_{14}\left(\pi_{\mathrm{u}}\right)$ & $709.1^{\mathrm{d}}$ & $162.2^{\mathrm{d}}$ & 619.7 & $159.8^{\mathrm{f}}$ & 621.5 & $122.1 \pm 24.4$ \\
\hline$v_{10}+v_{14}\left(\Sigma_{\mathrm{u}}^{+}\right)$ & $1265.93^{\mathrm{e}}$ & $66.1^{\mathrm{e}}$ & 1236.7 & $75.0^{\mathrm{f}}$ & 1229.7 & $63.0 \pm 12.6$ \\
\hline$v_{1}+v_{6}\left(\Sigma_{\mathrm{u}}^{+}\right)$ & $6523.9^{c}$ & $4.6^{\mathrm{c}}$ & 6550.8 & 5.7 & - & - \\
\hline
\end{tabular}

${ }^{a}$ Calculated values from ref. 17. ${ }^{b}$ Experimental values from ref. 16. ${ }^{c}$ Calculated values by the Local mode model with the single $\mathrm{CH}$ stretching displacement. ${ }^{\mathrm{d}}$ Calculated values by the normal mode model. ${ }^{\mathrm{e}}$ Calculated values by the Local mode model with the single $\mathrm{CCH}$ angle. ${ }^{\mathrm{f}}$ The value from Ref. 17 was multiplied by two for the degenerate $\pi_{\mathrm{u}}$ mode.

\section{(S4) Comparison of Rectilinear and Curvilinear Coordinate Systems}

The $M_{2}$ values derived using the linear response function in Table S2 were calculated by the curvilinear displacement coordinates (internal coordinates or bend angles) $\theta_{x}$ and $\theta_{y}$ of the CCH bending mode. Within the second order of the displacement coordinates, the $M_{2}$ values can be related to those by the rectilinear displacement coordinates (Cartesian coordinates) $X, Y$, and $Z$. In this section, this relationship will be clarified using the linearity of the linear response function.

As explained in Section 4.3 in the main text, the first derivatives of the external potential do not depend on whether curvilinear or rectilinear bending coordinate is used. From the discussion in eqs 28-30 and using the chain 
rule for partial derivatives, the second derivatives $v^{(2)}(\boldsymbol{r})$ in the curvilinear coordinates can relate to those in the rectilinear coordinates as follows:

$$
v^{(2)}(\boldsymbol{r})=\frac{1}{2} R_{\mathrm{CH}}^{2}\left\{\left[\frac{\partial^{2} v(\boldsymbol{r})}{\partial X^{2}}\right]_{X=0}+\left[\frac{\partial^{2} v(\boldsymbol{r})}{\partial Y^{2}}\right]_{Y=0}\right\}-R_{\mathrm{CH}}\left[\frac{\partial v(\boldsymbol{r})}{\partial Z}\right]_{Z=R_{\mathrm{CH}}}
$$

If the above right-hand expression is substituted in the linear response term in eq 26, and also the first-order relations $\partial v(\boldsymbol{r}) / \partial \theta_{x}=R_{\mathrm{CH}} \partial v(\boldsymbol{r}) / \partial X$ and $\partial v(\boldsymbol{r}) / \partial \theta_{y}=R_{\mathrm{CH}} \partial v(\boldsymbol{r}) / \partial Y$ in the nonlinear response term, then as a consequence of the linearity of $\chi\left(\boldsymbol{r}, \boldsymbol{r}^{\prime}\right)$, we can relate the $\sigma$ component of the second derivatives of $\rho(\boldsymbol{r})$ calculated with these different coordinate systems as follows:

$$
\rho^{(2)}(\boldsymbol{r})=\frac{1}{2} R_{\mathrm{CH}}^{2}\left\{\left[\frac{\partial^{2} \rho(\boldsymbol{r})}{\partial X^{2}}\right]_{X=0}+\left[\frac{\partial^{2} \rho(\boldsymbol{r})}{\partial Y^{2}}\right]_{Y=0}\right\}-R_{\mathrm{CH}}\left[\frac{\partial \rho(\boldsymbol{r})}{\partial Z}\right]_{Z=R_{\mathrm{CH}}}
$$

The two terms on the right-hand side of eq S5 are shown in Figure S1. Comparing these $\sigma$-symmetric components, the relation of eq S5 is interpreted as $\rho^{(2)}(\boldsymbol{r})$ in Figure 5c $=$ Figure S1b -Figure S1a, as was numerically verified. In particular, Figure S1a shows that the electron density derivatives on the left carbons are much smaller than those in Figure S1b, indicating that the perturbation caused by the $\mathrm{CH}$ stretching vibration along the z-axis is not easily transferred to the $\pi$-conjugated system. This corresponds well to the lower-lying ${ }^{1} \Sigma^{+}$ symmetry eigenvectors of the stability matrix (discussed in Section 4.5 and Table S7) do not contain any sizable $\sigma \rightarrow \sigma^{*}$ excitation components.

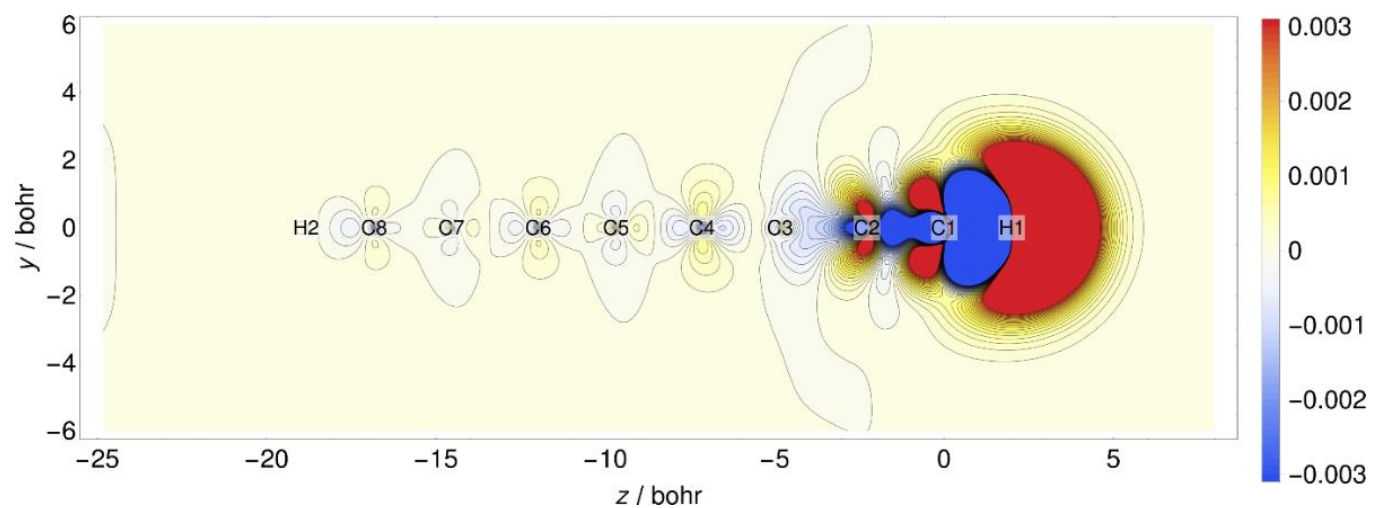

(a)

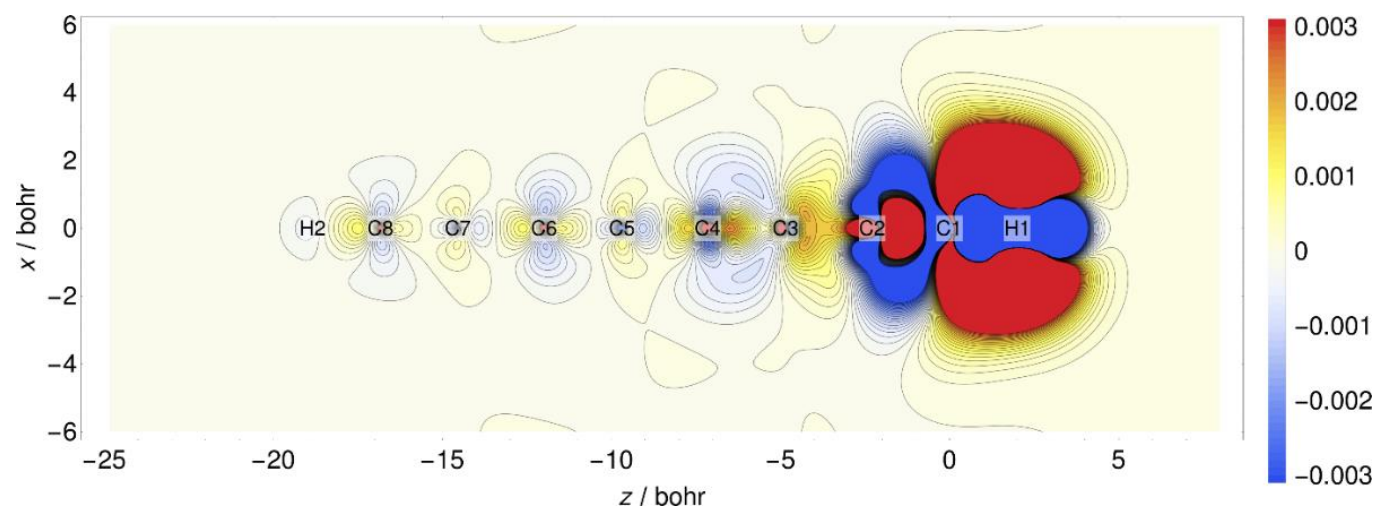

(b)

Figure S1. Electron density derivatives in eq $\mathrm{S} 5$ for $\mathrm{C}_{8} \mathrm{H}_{2}$. (a) $R_{\mathrm{CH}}(\partial \rho(\boldsymbol{r}) / \partial Z)_{Z=R_{\mathrm{CH}}}$ and (b) $\frac{1}{2} R_{\mathrm{CH}}^{2}\left\{\left[\frac{\partial^{2} \rho(r)}{\partial X^{2}}\right]_{X=0}+\left[\frac{\partial^{2} \rho(r)}{\partial Y^{2}}\right]_{Y=0}\right\}$ calculated by the rectilinear displacements. The bending $\mathrm{CCH}$ is placed at the right end. 
The value obtained by substituting the left-hand side of eq S5 into eq 18 should be the same as the sum of the values obtained by substituting the two terms on the right-hand side into eq 18 separately. The $M_{2}$ values calculated as the sum of these rectilinear displacement contributions in the $x, y$, and $z$ directions are shown in Table S5, where the nuclear contribution to $M_{2}$ is included as $\mathrm{Z}$ contribution. Reflecting the above discussion, the $M_{2}$ values in this Table S5 correspond well with the values from the linear response function shown in Table S2 above.

Table S5. The $M_{2}$ values of $\mathrm{C}_{2 n} \mathrm{H}_{2}$ calculated by the rectilinear vibrational coordinates by the linear response function (debye radian-2 $^{-2}$ ).

\begin{tabular}{|c|c|c|c|c|c|c|c|c|c|c|}
\hline Method & & $n=1$ & $n=2$ & $n=3$ & $n=4$ & $n=5$ & $n=6$ & $n=7$ & $n=8$ & $n=9$ \\
\hline \multirow{3}{*}{$\begin{array}{c}\text { Rectilinear } \\
\text { displacements }\end{array}$} & $M_{2}$ & -1.121 & -1.502 & -1.778 & -1.987 & -2.166 & -2.314 & -2.442 & -2.566 & -2.664 \\
\hline & $\begin{array}{c}X \text { and } Y \\
\text { contributions }^{\mathrm{a}}\end{array}$ & -0.671 & -0.912 & -1.102 & -1.239 & -1.359 & -1.457 & -1.542 & -1.626 & -1.693 \\
\hline & $\begin{array}{c}Z \\
\text { contribution }^{\mathrm{b}}\end{array}$ & -0.450 & -0.590 & -0.677 & -0.748 & -0.807 & -0.857 & -0.900 & -0.939 & -0.971 \\
\hline
\end{tabular}

The substitution of $v(\boldsymbol{r})$ in eq $\mathrm{S} 4$ by the potential energy functions $E\left(\theta_{x}, \theta_{y}\right) \equiv E(X, Y, Z)$ leads to a similar argument for the force constant of the local CCH bending mode. In this case, by definition, $\left[\frac{\partial E(X, Y, Z)}{\partial Z}\right]_{Z=R_{\mathrm{CH}}}=0$ in the equilibrium structure, so the bending frequency does not depend on the curvilinear or rectilinear displacement within the harmonic approximation. If the local $\mathrm{CCH}$ bending mode is expressed using the rectilinear displacement $X$, the contribution of the displacement in the $z$-direction is neglected in the second term on the right-hand side of S5. As shown in Table S5, this contribution of $\partial \rho(\boldsymbol{r}) / \partial Z$ has a magnitude of about 35-40\% of $M_{2}$, thus considering only $\partial^{2} \rho(\boldsymbol{r}) / \partial X^{2}$ will result in a large error, unless configuration mixings between the overtone bending wavefunctions in the $x$ and $y$ directions and the fundamental stretching in the $\mathrm{z}$-direction are accounted for by including the anharmonic couplings. 


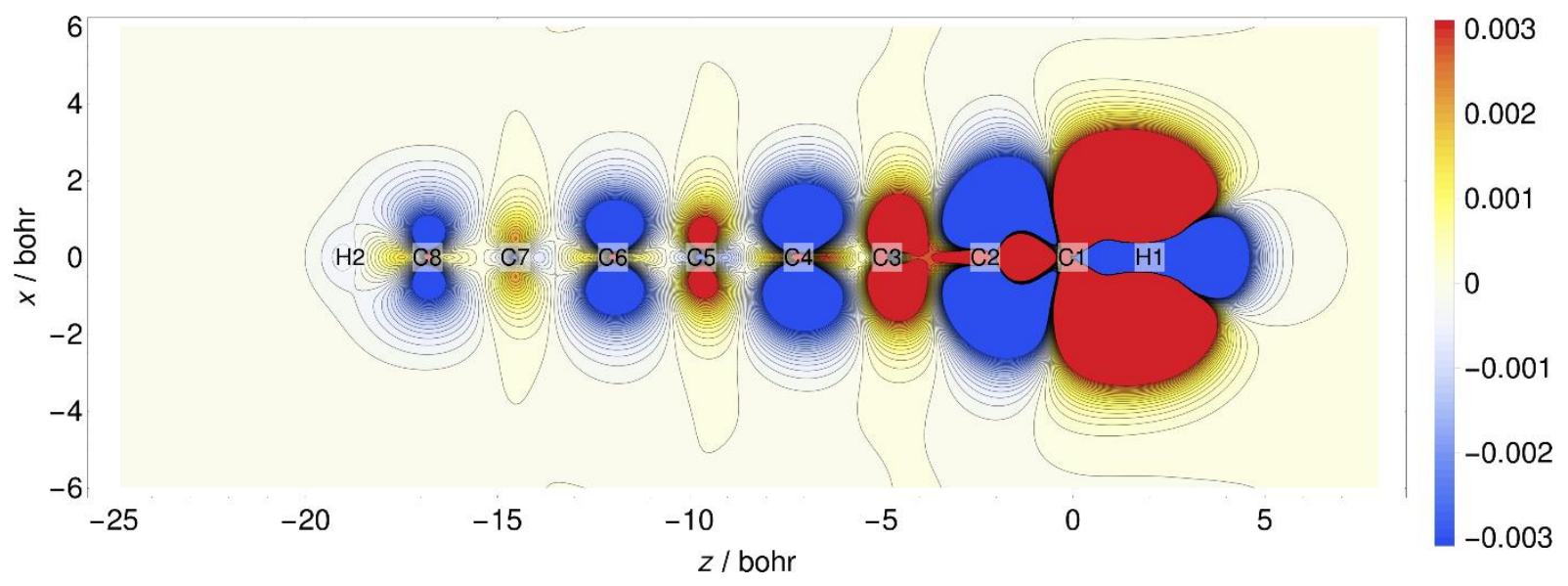

(a)

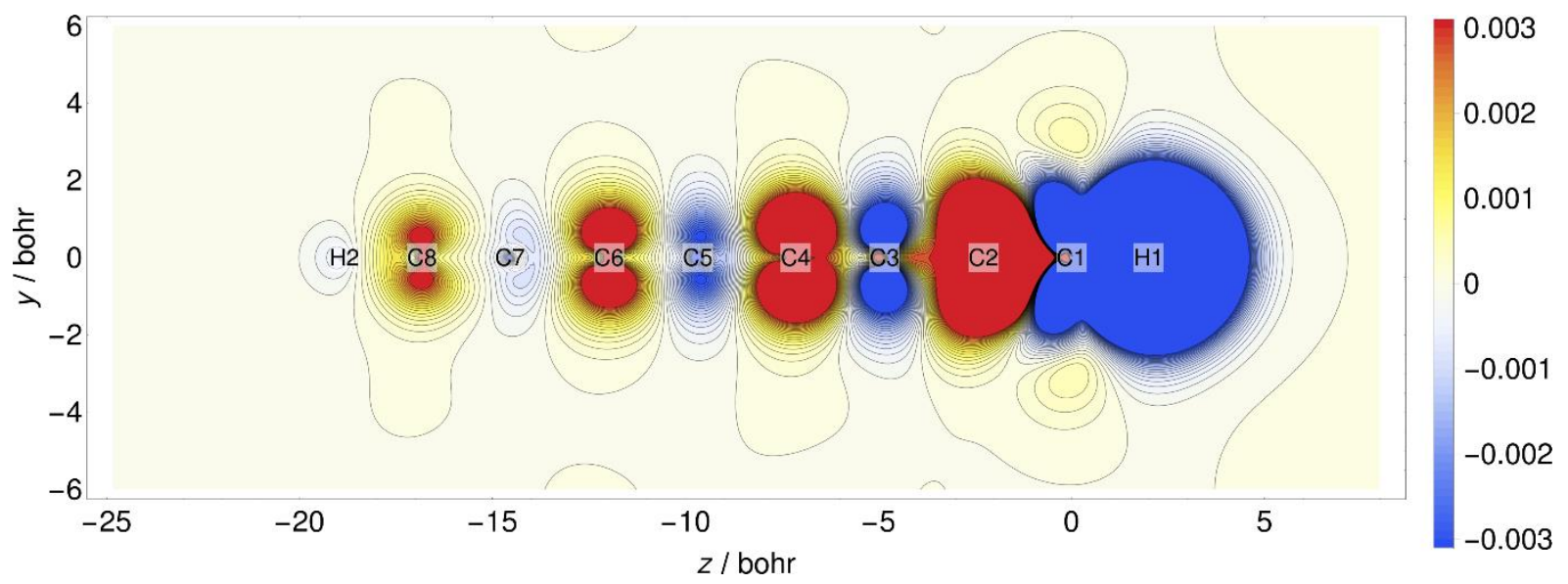

(b)

Figure S2. Electron density second derivatives with respect to the bond angle $\theta_{x}$ of the $\mathrm{H} 1$ atom $\left(\partial^{2} \rho(\boldsymbol{r}) / \partial \theta_{x}{ }^{2}\right)$ for $\mathrm{C}_{8} \mathrm{H}_{2}$ calculated by the linear response function. The bending $\mathrm{CCH}$ is placed at the right end. (a) and (b) show the contour maps in the xz and yz plane, respectively. The values in these contour plots are identical as those in the isosurfaces in Figure $5 \mathrm{~b}$. 


\section{(S6) Mutual Polarizability for the Long Polyyne}

In Section 4.4 we have discussed that the $\pi$ electron density changes of $\mathrm{C}_{8} \mathrm{H}_{2}$ can be reproduced in the sumover-state representation including only four MOs with two symmetric and two antisymmetric orbitals. Here we will see if this feature also holds for longer polyynes such as $\mathrm{C}_{40} \mathrm{H}_{2}$, which has $20^{2}=400$ possible excitations with a combination of occupied and unoccupied orbitals. The lowest 10 excitations in ascending order of the excitation energy $\varepsilon_{a}-\varepsilon_{i}$ are shown in Table S6. The four excitations in eq 46 discussed for $\mathrm{C}_{8} \mathrm{H}_{2}$ corresponds to truncation by the sum of the contributions of the excitations with $m=1,2,3$, and 6 .

Table S6. The excitation energy of Hückel orbital for $\mathrm{C}_{20} \mathrm{H}_{2}$.

\begin{tabular}{cc|l|c}
\hline Rank $m$ & $(i, a)$ & Assignment & Excitation energy $(-\beta$ unit $)$ \\
\hline 1 & $(20,21)$ & HOMO $\rightarrow$ LUMO & 0.153211 \\
2 & $(20,22)$ & HOMO $\rightarrow$ LUMO+1 & 0.305972 \\
3 & $(19,21)$ & HOMO-1 $\rightarrow$ LUMO & 0.305972 \\
4 & $(20,23)$ & HOMO $\rightarrow$ LUMO+2 & 0.457388 \\
5 & $(18,21)$ & HOMO-2 $\rightarrow$ LUMO & 0.457388 \\
6 & $(19,22)$ & HOMO-1 $\rightarrow$ LUMO+1 & 0.458734 \\
7 & $(20,24)$ & HOMO $\rightarrow$ LUMO+3 & 0.606568 \\
8 & $(17,21)$ & HOMO-3 $\rightarrow$ LOUMO & 0.606568 \\
9 & $(19,23)$ & HOMO-1 $\rightarrow$ LUMO+2 & 0.610149 \\
10 & $(18,22)$ & HOMO-2 $\rightarrow$ LUMO+1 & 0.610149 \\
\hline
\end{tabular}

The mutual polarizability truncated by the $m$ th excitation $-\pi_{p, 1}^{m}$ is calculated based on eq 42 and shown in Figure S3. As shown in Figure S3a, the absolute value of $-\pi_{p, 1}^{m}$ for C1 and C2 is underestimated in the case of $m=1,2,3,6$. Since their values of C1 and C2 become rather well reproduced by the sum of excitations with $m \leq 200$, they exhibit a slow convergence on the carbon atoms around the bending $\mathrm{CCH}$. This feature reminiscent that the Dirac delta function consists of a wider range of wavelength components. On the other hand, the decay of the amplitude of the $\pi$ electron density changes on distant carbon atoms from the bending $\mathrm{CCH}$ and their sign alternations there are well reproduced by the sum of $m=1,2,3,6$ excitations, which include only two symmetric and two antisymmetric excitations. It is noted in Figure S3b that, $-\pi_{p, 1}^{m}$ truncated at $m \leq 10$ shows the negative sign on both the odd- and even-numbered carbon atoms on the left-hand side, and cannot correctly reproduce the sign alternations. This implies the importance of balanced phase cancellations in these distant carbon regions, where the magnitude of the small $\pi$-electron density changes determines the gradual size dependence of the $M_{2}$ values, thus the absorption intensities. 


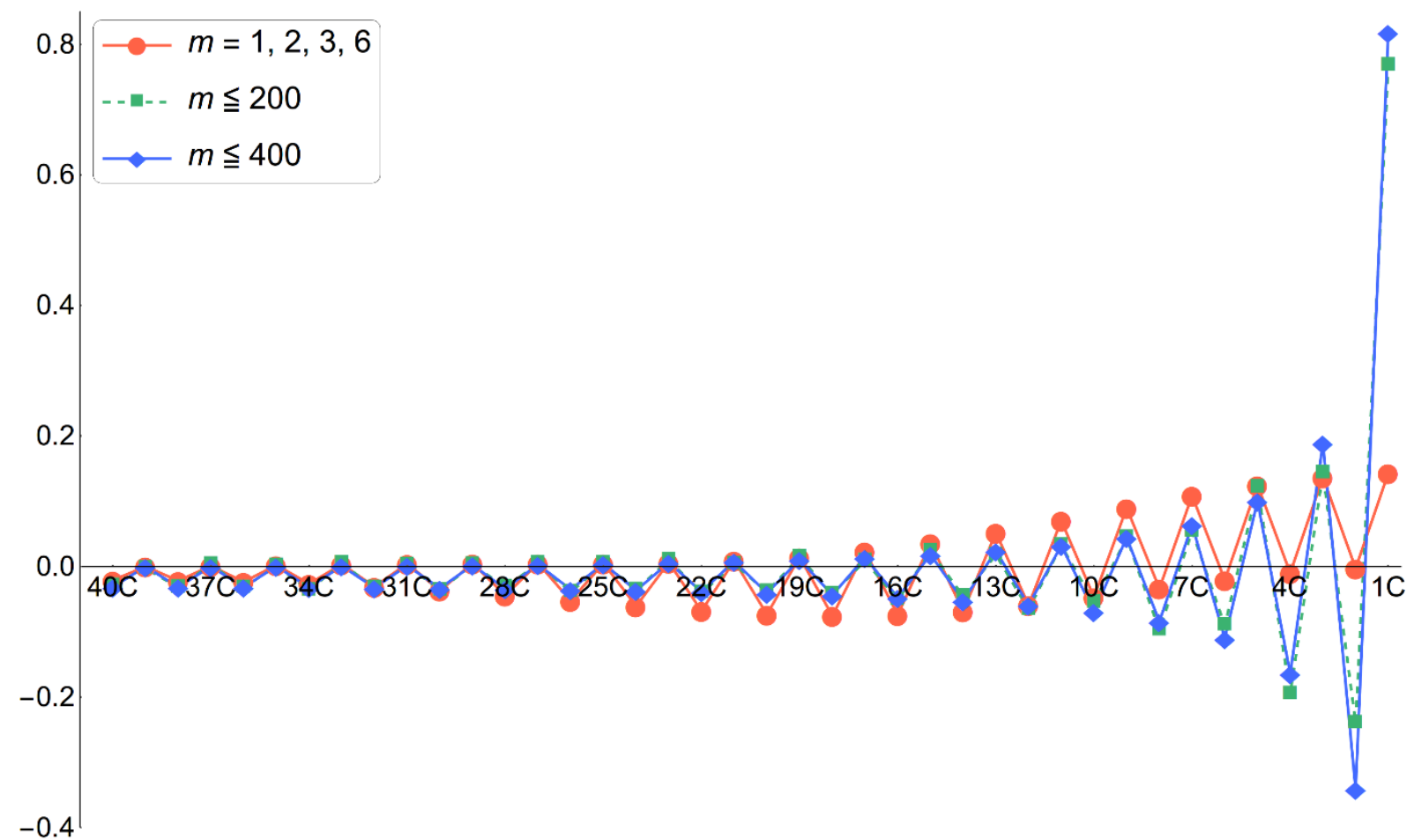

(a)

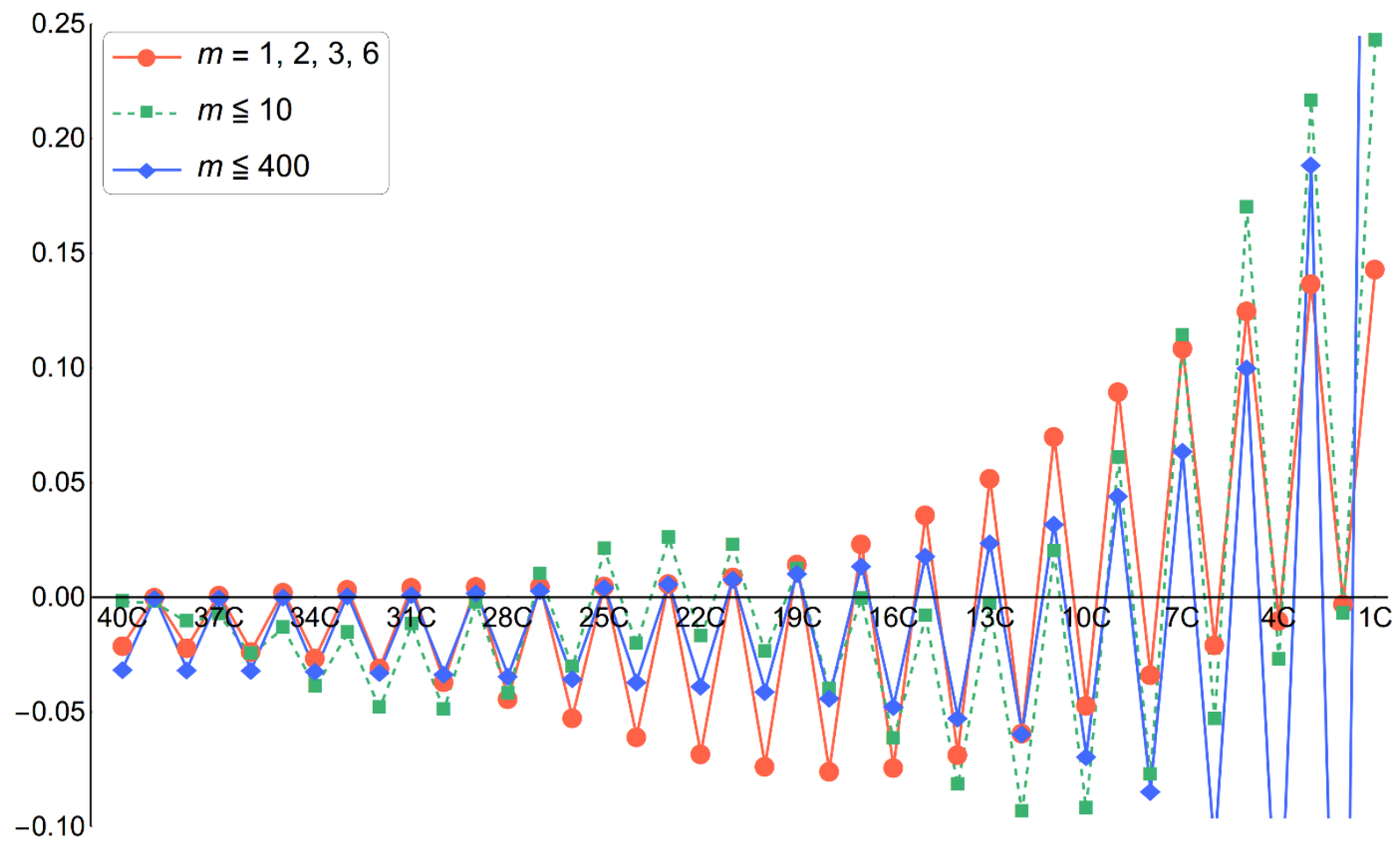

(b)

Figure S3. The mutual polarizability $-\pi_{p, 1}^{m}$ truncated at the lowest $m$ th excitation for $\mathrm{C}_{40} \mathrm{H}_{2}$. (a) shows the sum of $m=1,2,3,6$ (excitations in eq 46 ), $m \leqq 200$, and $m \leqq 400$ (all excitations). (b) shows the sum of $m=1,2,3,6$ (excitations in eq 46 ), $m \leqq 10$, and $m \leqq 400$ (all excitations). 
(S7) Instability Eigenvalues

Table S7. Eigenvectors and eigenvalues (hartree) of the stability matrix for the linear $\mathrm{C}_{8} \mathrm{H}_{2}$.

\begin{tabular}{lcc}
\hline \multicolumn{1}{c}{ Dominant character } & Symmetry & Eigenvalue \\
\hline $\mathrm{HOMO} \rightarrow$ LUMO & ${ }^{1} \Delta_{\mathrm{u}}$ & 0.1157965 \\
\hline $\mathrm{HOMO}-1 \rightarrow$ LUMO and minor $(\mathrm{HOMO} \rightarrow$ LUMO +1$)$ & ${ }^{1} \Sigma_{\mathrm{u}}^{+}$ & 0.2817158 \\
\hline $\mathrm{HOMO} \rightarrow \sigma_{\mathrm{g}}{ }^{*}$ & ${ }^{1} \Sigma_{\mathrm{g}}^{+}$ & 0.1856768 \\
\hline $\mathrm{HOMO} \rightarrow \mathrm{LUMO}+1$ and minor $(\mathrm{HOMO}-1 \rightarrow$ LUMO) & ${ }^{1} \Delta_{\mathrm{g}}$ & 0.2534869 \\
\hline${ }^{1} \Gamma_{\mathrm{g}}{ }_{\mathrm{g}}$ & 0.2443675 \\
\hline $\mathrm{HOMO}-2 \rightarrow \mathrm{LUMO}$ & ${ }^{1} \Delta_{\mathrm{u}}$ & 0.3084083 \\
\hline $\mathrm{HOMO} \rightarrow \sigma_{\mathrm{u}}{ }^{*}$ & ${ }^{1} \Sigma^{+}{ }_{\mathrm{u}}$ & 0.3158962 \\
\hline $\mathrm{HOMO} \rightarrow \sigma_{\mathrm{g}}{ }^{*}$ & ${ }^{1} \Pi_{\mathrm{u}}$ & 0.2547161 \\
\hline
\end{tabular}

